\title{
Intelligent Controlling System of Aquiculture Environment
}

\author{
Deshen Zhao,"* and Xuemei $\mathrm{Hu}^{2}$ \\ ${ }^{1}$ Department of Electric Engineering, Henan Polytechnic Institute, Nanyang, \\ Henan Province, P.R. China 473009, \\ ${ }^{2}$ Department of Electrical and Mechanical Engineering, Henan Polytechnic Institute, Nanyang, \\ Henan Province, P.R. China 473009, \\ Tel.: +86-0377-632769922; Fax: +860-377-63270216 \\ zhaodesh@163.com
}

\begin{abstract}
The paper has analyzed present aquiculture conditions and controlling problems of water environment factors of aquiculture, and constructed effective security aquiculture breeding intelligence controlling system suitable to Chinese situation, and presented the control strategy of neural network realizing dynamic decoupling for the factory aquiculture, and specially solved the water environment control and so on the key questions. The long term practice has shown that the system operation is simple and effective safe by applying some breeding bases in Zhenjiang, the system has met the requirements of culturists and enhanced international market competition for aquiculture.
\end{abstract}

Keywords: Aquiculture environment factors, Fuzzy control, Neural network, Decoupling control.

\section{Introduction}

Aquiculture environment is a complex system engineering which affects growth process of aquatic animals and plants, there are many questions of technology, information, management and aquaculture environmental control and other issues, the application of high technology is relatively weak. These won't make culturists take timely measures when they encounter the problems, which will delay the best growth time of plants and animals, and cause serious losses. It is the key for efficient application of water resources and technology integration to achieve efficient aquatic product, China's current aquaculture management has changed from mainly relying on the experiences to decisions of the scientific decisions, these can make rapid response and timely decision according to the status changes of aquatic animals and plants resources and guarantee the prevalence of efficient using resources model. The study will associate aquaculture expert technical knowledge, scientific research and long term practical experience of culturists with computer technology and fieldbus technology and establish a comprehensive, intelligent environment controlling system which will stead for the growth of aquatic plants and animals.

\footnotetext{
${ }^{*}$ Corresponding author.
} 


\section{System Hardware Structure}

Controlling system of aquiculture environment regulates environmental temperature, dissolved oxygen, PH, and salinity such as breeding environmental factors by controlling furnishments and artificially creates their growth environment according to their different growth phases, the system detects real-time parameters of breeding environmental and automatically adjusts states of the various controlling equipment after comparing the measured parameters and the standard parameters of system configuration in order to meet aquiculture growth requirements to the various environmental factors.

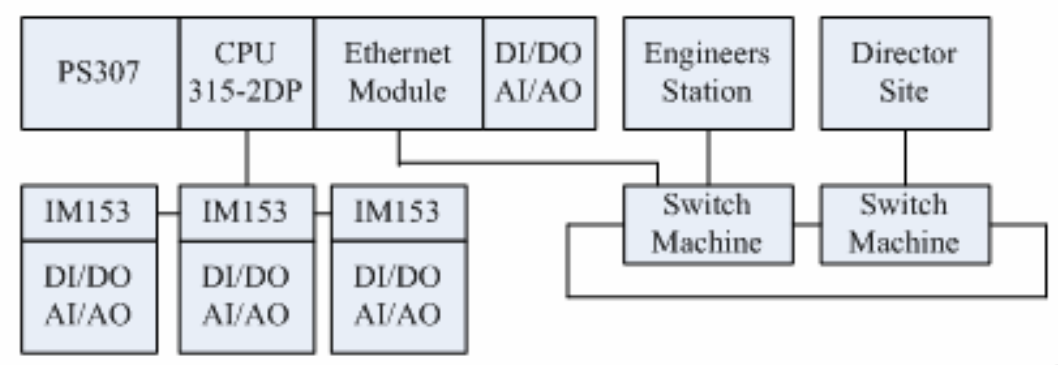

Fig. 1. System hardware structure

System hardware (Ma Congguo, et al, 2005) includes three components: 1) signal acquisition: including temperature, dissolved oxygen, $\mathrm{PH}$, as well as salinity detection environmental factors. 2) Signal conversion and processing: Real-time data acquisition signals will be transformed to the digital and analog. 3) Output and controlling parts: speed of the machine of increasing oxygen and fan and opening of windows and electromagnetism valve, The system hardware is composed of monitoring computer and PROFIBUS-DP field controlling units, which detects and controls the ecological environment factors through numeric and analogy input and output modules of remote I/O controlling units, the PLC ,engineers stations and director Room can monitor the states of controlling furnishments and the ecological breeding environment. The system consists of three controlling modules, the overall framework as shown in Fig.1.

\section{System Software Structure}

The whole system software includes the PLC monitoring units software and computer monitoring software, the development software of field monitoring Unit PLC uses STEP7-Micro/WIN32; monitoring computer software adopts Siemens Wincc6.0; production management software applies Borland $\mathrm{C}++$ Builder6.0, the system software structure includes: (1) PLC software: PLC program can be divided into three modules: (1) Data acquisition modules sample the pretreatment sensor data of aquiculture breeding environment, (2) Environmental parameters controlling modules control the states of controlling furnishments according to the error between system configuration and the actual value of sensor detection, (3)Communication modules are mainly responsible for PLC and the I / O modules, monitoring computer communication. 
(2) Monitoring software layer: monitoring computer software is operating platform of controlling breeding environment process, program includes four modules: (1) Manmachine interface displays real-time data, regular store data, generating controlling commands of breeding process, (2) Data management module defines four databases of the system: real-time database, technology database, task database, operating log database, (3) The system management module mainly answers for the system equipment failure, prediction ,diagnosis, guidance etc, (4) Data processing module mainly inquires about historical data and processes data. (3) Production management software: production management computer software provides user interface of different information management, which includes the following modules: (1)User management (2) human-machine interface (3) Data query (4) system status.

\section{Controlling Algorithm}

The aquatic product environment has many factors, such as temperature, dissolved oxygen, PH value, water level and so on, because the temperature and dissolution is the most important 2 environment factors for the aquatic product benefit, the paper has taken the temperature and dissolved oxygen as example, and regulated controlling equipment states to meet system request, the input of fuzzy controller (Hao jiu Yu,et al, 2004)is erroneous and erroneous change rate, the output of fuzzy controller is neural network input, the output of neural network controls equipment states. The change process of the dissolved oxygen and temperature of aquiculture environment is big inertia, time variable, nonlinear, strong coupling multi-inputs and outputs system ( $\mathrm{Li}$ $\mathrm{Xu}$ Ming, 2001), the intelligent controller based on neural network decoupling has controlled aquatic product environment, and combined breeding expert experience with fuzzy control and neural network technology. It utilizes humanity experience knowledge, fuzzy logic inference and neural network study to solve the control strategy which adapted cultivation environment, and applies online study to seek its decoupling relations by using BP algorithm, this algorithm can realize the static and dynamic decoupling, fuzzy neural network decoupling control system is shown in Fig. 2.

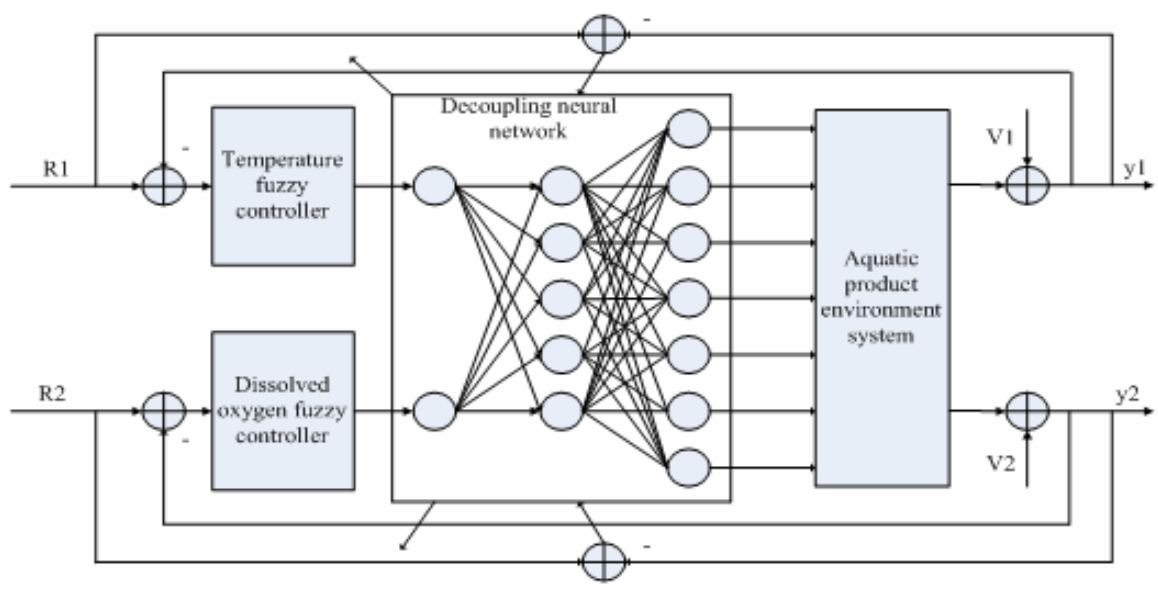

Fig. 2. Decoupling controller of aquiculture environment 
Among: $\mathrm{R} 1$ and $\mathrm{y} 1$ as the temperature setting value and actual value, $\mathrm{R} 2$ and $\mathrm{y} 2$ as the dissolved oxygen setting value and actual value. The decoupling algorithm is composed of the fuzzy controller and neural network, the neural network is trained by off line, it will gradually adapt environment change through the study and undertakes the controlling duty; when the cultivation environment is disturbed or the parameters change suddenly, the parameter performance can't satisfy the request, the fuzzy controller and neural network will adjust proportionality factors of fuzzy controller and neural network weight to enable the control system to adapt the aquatic product growth environment.

\subsection{Fuzzy Controller Design}

A fuzzy regulator using self-adjustment factor consists of two parallel component parts of fuzzy control and integral role, its fuzzy control rules:

$$
U f=K 0 * f\left(e, e^{\prime}\right)
$$

Among formula: $U f$ as fuzzy regulator output, $K O$ as output coefficient, $f\left(e, e^{\prime}\right)=\alpha * e+(1-\alpha) * e^{\prime} . \alpha$ for adaptive correction factor, $0 \leq \alpha \leq 1 ; \alpha$ reflects the size of the error e and error rate e' on impact of the regulator output, at the initial stage, the main tasks of the control eliminates error, Therefore, $\alpha$ value should be greater than 0.5 ; when the system is close to stable, and should avoid oscillation, $\alpha$ value should be less than 0.5 , this paper takes to 0.7 and 0.3 .

\subsection{Neural Network Design}

Neural network (Li Ming, 2000) is 3 layers BP networks of 2 input nodes, 5 latent nodes and 7 output nodes, this first layer of this networks is the output of the fuzzy controller, the output of the third layer is correspondence controlling equipment states, this layer uses sigmoid activation function, the output controlling quantity of decoupling neural network is limited between $0-1$, and is multiplied by suitable output factor to control external equipment. To switch quantity controlling equipments, when the output is bigger than 0.5, correspondence equipment will open, or it will close; To shift quantity controlling equipments, when the output is bigger than 3 or 2 or 1 , it will be high or medium or low speed,or it will stop. The computational method of decoupling BP neural network includes forward and counter algorithm. The forward formula of neural network is following: the input layer has two nodes, they correspond the output of fuzzy controller of the temperature and dissolved oxygen, its input and output function is:

$$
O_{i}^{\prime}(k)=x_{i}^{\prime}(k)
$$

Among: $\boldsymbol{X}_{i}^{\prime}(k)$ as the neuron value of the input layer of $\mathrm{k}$ sampling time, $\boldsymbol{O}_{i}(k)$ as the output neuron value of the input layer of $\mathrm{k}$ sampling time, $\mathrm{i}$ as neuron number, $i=1,2$. Latent layer have 5 neurons, the neuron value is the weight sum of the output of the corresponding input layer neuron, and the formula is:

$$
x_{j}^{\prime \prime}(k)=\sum_{i=1}^{2} w_{i j}^{\prime \prime}(k) \boldsymbol{O}_{i}^{\prime \prime}(k)
$$


The activation function of latent layer takes the positive and negative symmetrical Sigmoid function, among formula: $w_{i j}^{\prime \prime}(k)$ as connection weight of input layer to latent layer, $\boldsymbol{x}_{i}{ }^{\prime}(k)$ as the output value of latent layer neuron, $\boldsymbol{O}_{i}{ }^{\prime}(k)$ as the input value of latent layer neuron, $I=1,2, j=1,2,3,4,5$. The output layer has 7 nodes, they correspond 7 controlling equipments, the input of each neuron is the weight sum of the output of latent layer neuron, and the formula is:

$$
\boldsymbol{X}_{o}^{\prime \prime \prime}(k)=\sum_{i=1}^{5} \boldsymbol{W}_{j o}^{\prime \prime \prime} \cdot \boldsymbol{O}_{j}^{\prime \prime}(k)
$$

Because the latent layer completely connects the output layer, during the latent layer mapping the output layer process, neural network has realized decoupling of overall system, and simultaneously sent the most superior decoupling results for the external controlling equipments, the nodes of the output layer select nonnegative sigmoid activation function.

\subsection{Counter Algorithm of Decoupling Network}

Learning rule of BP neural network (Sun Xin ,2005) is one kind of Delta rule, and adjusts connection weight by using error antigradient,and makes the outlet error reduce monotonously, the counter algorithm regards the decoupling network and fuzzy regulator as well as the multivariable controlled object as multi-layer generalizing network, the multivariable controlled object is the final layer of this network, the learning algorithm of erroneous reversion adjusts error, the system has considered various variables coupling function, and taken performance target function [4] is:

$$
J=E(k)=\frac{1}{2} \sum_{s=1}^{2}\left(r_{S}(k)-y_{S}(k)\right)^{2}
$$

According to the gradient descent law modifying coefficient of network weights, antigradient direction of $\mathrm{E}(\mathrm{k})$ to weighting coefficient searches adjustment, and attaches inertia item to make the rapid convergence to overall minimum, and obtains adjustment formula (Wang Da Yi,1999) of the output layer network weight:

$$
w_{j o}^{\prime \prime \prime}(k)=w_{j o}^{\prime \prime \prime}(k-1)+\Delta w_{j o}^{\prime \prime \prime}(k)
$$

According to similar principle, connection weight iterative formula of input layer to latent layer is:

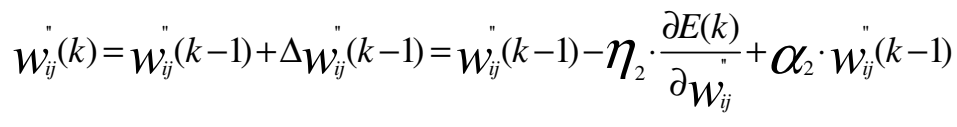

Controlled object doesn't need mathematical model and the parameter according to the neural network algorithm, the system trains BP network by off line, it may be put into operation of the systems after training, the connection weight adjustment formula reference (6), (7). 


\subsection{Controlling Results}

The decoupling system of fuzzy neural network has controlled aquatic product environment factors, this control system of neural network decoupling has solved both temperature and dissolve coupling function, and considered controlling quality low consequence of controlling equipment frequent start-up, and improved the controlling quality. The long term application has indicated neural network decoupling controller has obtained good controlling effect., Year trial and observation have shown that the system can maintain appropriate growth environment, when it is applied to $20 \mathrm{~m} \times$ $20 \mathrm{~m}$ the breeding ponds. We may see change situation of breeding pond temperature and dissolved oxygen from the next table, the starting value of temperature and dissolved oxygen is $30^{\circ} \mathrm{C}$ and $7.5 \mathrm{mg} / \mathrm{L}$, the actual value and given value are compared, the errors exist in $2 \%$, and meets the system growth requirement, the controlled process doesn't basically have over modulation, the adjustment time is short, the stable errors doesn't basically have.

For example: the original temperature to $32^{\circ} \mathrm{C}$, dissolved oxygen to $7.15 \mathrm{mg} / \mathrm{L}$, controlling equipment are all closed, they begin to control according to the detection of the errors and decoupling, the system sets temperature at $25^{\circ} \mathrm{C}$ and dissolved oxygen at $7.5 \mathrm{mg} / \mathrm{L}$, The sampling period is $20 \mathrm{~s}$ and the equipments have the same controlling state within 6 minutes, The state changes of controlling equipments are shown in table 1, among table: 0-3 representatives of the stall controlling, they represent off or stop, small or low-speed, medium or medium-speed, open or high-speed.

We can see the temperature will drop to $29.0^{\circ} \mathrm{C}$ and dissolved oxygen will rise to 7.25 in 6 minutes from table 1, under the new deviation, this opening of controlling equipments are changed $(0,1,1,1,0,2,2)$, in another 6 minutes, the temperature will drop to $26.5^{\circ} \mathrm{C}$ and dissolved oxygen will rise to 7.38 , and the opening is $(0,0,0,1,0,1,1)$ etc. The system will become small deviation in 20 minutes. At different initial value of temperature and dissolved oxygen, Experiments have been applied and the results are very good.

Table 1. Aquiculture environment control results

\begin{tabular}{lllllllllll}
\hline $\mathrm{t}(\mathrm{M})$ & $\mathrm{T}\left({ }^{\circ} \mathrm{C}\right)$ & $\mathrm{D}(\mathrm{m} \mathrm{g} / \mathrm{L})$ & $\mathrm{U}$ & $\mathrm{S}$ & $\mathrm{N}$ & $\mathrm{C}$ & $\mathrm{H}$ & $\mathrm{F}$ & $\mathrm{M}$ \\
\hline 1 & 31.5 & 7.15 & 1 & 2 & 2 & 2 & 0 & 2 & 3 \\
2 & 30.0 & 7.20 & 1 & 2 & 2 & 2 & 0 & 2 & 3 \\
4 & 29.0 & 7.25 & 1 & 2 & 2 & 2 & 0 & 2 & 3 \\
6 & 28.0 & 7.30 & 1 & 2 & 2 & 2 & 0 & 2 & 3 \\
8 & 27.0 & 7.35 & 0 & 1 & 1 & 1 & 0 & 2 & 2 \\
10 & 26.5 & 7.38 & 0 & 1 & 1 & 1 & 0 & 2 & 2 \\
12 & 26.0 & 7.40 & 0 & 1 & 1 & 1 & 1 & 2 & 2 \\
14 & 25.7 & 7.45 & 0 & 0 & 0 & 1 & 0 & 1 & 1 \\
16 & 25.3 & 7.48 & 0 & 0 & 0 & 1 & 0 & 1 & 1 \\
18 & 25.1 & 7.50 & 0 & 0 & 0 & 1 & 0 & 1 & 1 \\
20 & 24.9 & 7.49 & 0 & 0 & 0 & 1 & 0 & 1 & 0 \\
\hline
\end{tabular}

Among table: t- controlling time, T-temperature, D-Dissolved oxygen, U-sunroof, S-south window, $\mathrm{N}$-north window, C- cold water valve, H-hot water valve, F-fan,M- increasing oxygen machine. 


\section{Conclusion}

The method of fuzzy neural decoupling has improved controlling level of aquiculture breeding environment and taken on the larger theoretical and practical significance, real-time testing results have demonstrated its feasibility, and it has greatly practical value. With the existing pattern compared to this controlling scheme, the system is open and achieves data sharing, thus has enhanced the level of controlling breeding environment. Intelligent decoupling control system of the aquatic product environment has strong comprehensive, usable, scientific and compatibility to breeding environment, the system needs aquaculture expert and engineering technology intelligence expert mutual coordination, and closely unifies the domain knowledge experience and project technology, the practice has shown the system has enhanced cultivation benefit, and improved aquatic growth environment.

\section{References}

Congguo, M., Wei, N.: Factorization aquaculture monitoring system design based on PLC. Industry appliance and automation device 12(2), 51-53 (2005)

Yu, H.j., Wei, C.: Decoupling for multivariable fuzzy Control System. Tianjin University Journal 37(5), 396-399 (2004)

Ming, L.X.: Fuzzy neural network control of multivariable system. Control and Decision 16(1), 107-110 (2001)

Ming, L., Yongjun, L.: Auto-adapted neuron non-model much variable system decoupling control. Computer simulation (3), 68-71 (2000)

Xin, S.: Quota moisture decoupling control analyzes of ma paper product process. Control theory and application 20(18), 121-124 (2005)

Yi, W.D., Rui, M.X.: Neuro-Optimal Guidance Law for Lunar Soft Landing. Engineering and Electronic Technology 21(12), 31-34 (1999) 